\title{
Three-dimensional structure from motion recovery of a moving object with noisy measurement
}

\author{
Zoubaida Mejri ${ }^{1}$, Lilia Sidhom ${ }^{2}$, Afef Abdelkrim ${ }^{3}$ \\ ${ }^{1,2,3}$ Research Laboratory L.A.R.A in Automatic control, National Engineering School of Tunis (ENIT), \\ University of Tunis El Manar, Tunisia \\ ${ }^{1,3}$ National Engineering School of Carthage (ENICarthage), University of Carthage, Tunisia \\ ${ }^{1,2}$ The National Engineering School of Bizerte (ENIB), University of Carthage, Tunisia
}

\begin{tabular}{|c|c|}
\hline Article Info & ABSTRACT \\
\hline Article history: & In this paper, a Nonlinear Unknown Input Observer (NLUIO) based \\
\hline Received Mei 3, 2019 & $\begin{array}{l}\text { approach is proposed for three-dimensional (3-D) structure from motion } \\
\text { identification. Unlike the previous studies that require prior knowledge of }\end{array}$ \\
\hline Revised Aug 8, 2019 & either the motion parameters or scene geometry, the proposed approach \\
\hline Accepted Aug 29, 2019 & $\begin{array}{l}\text { assumes that the object motion is imperfectly known and considered as an } \\
\text { unknown input to the perspective dynamical system. The reconstruction of }\end{array}$ \\
\hline Keywords: & $\begin{array}{l}\text { the 3-D structure of the moving objects can be achieved using just two- } \\
\text { dimensional (2-D) images of a monocular vision system. The proposed }\end{array}$ \\
\hline $\begin{array}{l}\text { Measurement noise } \\
\text { Monocular vision systems }\end{array}$ & $\begin{array}{l}\text { scheme is illustrated with a numerical example in the presence of } \\
\text { measurement noise for both static and dynamic scenes. Those results are }\end{array}$ \\
\hline $\begin{array}{l}\text { Nonlinear unknown input } \\
\text { observer }\end{array}$ & used to clearly demonstrate the advantages of the proposed NLUIO. \\
\hline Structure from motion & $\begin{array}{r}\text { Copyright (C) } 2020 \text { Institute of Advanced Engineering and Science. } \\
\text { All rights reserved. }\end{array}$ \\
\hline \multicolumn{2}{|l|}{ Corresponding Author: } \\
\hline $\begin{array}{l}\text { Zoubaida Mejri, } \\
\text { Department of Electrical E } \\
\text { National Engineering Scho } \\
\text { University of Carthage, } 45 \\
\text { Email: zoubaida.mejri@en }\end{array}$ & $\begin{array}{l}\text { age (ENICarthage), } \\
\text { trepreneurs, Charguia II, 2035. Tunis, Tunisia. }\end{array}$ \\
\hline
\end{tabular}

\section{INTRODUCTION}

The progress in structure and motion estimation (a.k.a. structure-from-motion) research has been hectic, stimulated by recent breakthroughs in computer vision, the advent of digital photography and the augmented reality [1-6]. This progress has the potential to substantially increase the use of the structure from motion technique for a variety of applications, for example the growing application of unmanned aerial vehicles for remote surveying for a numerous of ecological domain [7]. Wide-reaching marine assessments using this technique have recently become possible in some cases like in [8,9] with drone-based application. The structure from motion technique can be used for topographic data collection in field and laboratory studies [10] and as a means of digital preservation and for documenting archaeological excavations, cultural material and architecture [11]. On the other side, structure from motion can be a good low-cost alternative to generate high resolution topography [12], where light detection and ranging data is unaffordable or scarce. Recently in the area of agriculture [13], the use of unmanned aerial systems (UAS) based on the structure from motion technique as remote-sensing platforms have massive potential for obtaining detailed of crop features. The structure and motion field of research is worried with the recovery of 3-D geometry of the dynamic scene (the structure) when observed through a moving camera (the motion). Basically, structure from motion involves three main steps: First extracting features in images and matching these features between images, then modeling the camera-object relative motion and finally recovery of the 3-D structure using the estimated motion and features. 
Keeping in view the above literature, several works have addressed structure estimation observer based approach where full velocity parameters feedback of the calibrated camera was provided. Such as in [14] where authors designed a nonlinear observer to estimate an unmeasurable state called depth with known dynamics. That last one has been experimented on a mobile robot with an on-board camera. Authors in [15] have introduced a nonlinear observer for a particular case of feature points on the object moving with constant velocities and have approved in many practical scenarios. Although, in [16] a nonlinear observer is defined to recover structure and motion with less restrictive assumptions on the moving object motion. A reduced-order nonlinear observer is presented in [17] to estimate the range from a moving camera to a feature point on a static scene. Furthermore, a design of complete order observers based on nonlinear contraction theory and synchronization is given in [18] where angular and linear velocity measurements are also noisy.

The information of the camera motion parameters has been unavoidable in the preceding cited references. Various studies on structure from motion estimation are also available where the camera motion is not known. Starting with [19], sliding mode observers were presented to estimate the motion parameters and the structure of a moving object with the aid of a change-coupled device (CCD) camera. The advantage presented by the proposed observers is that both rigid and affine motion parameters, constant or timevarying, can be estimated correctly. In the uniqueness context, [20] introduced a developed nonlinear reduced order observer which only requires one camera linear velocity to estimate a stationary object seen by a calibrated camera. The methods described in [21, 22] present nonlinear observers based on a Robust Integral Signed Error method (RISE) to estimate the unknown distance between the camera and the object and the moving camera velocities. This problem was also investigated in [23] where a nonlinear reducedorder observer is proposed to recover the feature point depth and camera linear velocity. Only the camera's angular velocity is assumed to be known. Authors described in [24] a new approach based on Extended Kalman Filter to simultaneously recover camera pose and the structure of non-rigid extensible surfaces. In order to extend the problem to a deformable domain, authors defined the object's surface mechanics by means of Navier's equations. A recent paper [25] addresses the case where a novel complete-order observer is designed to estimate the unknown motion parameters and feature depth in the presence of measurement noise. The observer is derived from a differentiator based on the sliding-mode technique.

This paper, tackles the problem of motion and structure recovery for a class of system consisted on a moving camera moving object. Naturally, motions are constructed in continuous time settings and the motion parameters are assumed to be all time varying. The 3D position is estimated by using a set of image data observed through a dynamic camera with varying focal length. The contributions of this paper are first the analysis of the extent to which a scheme can be developed that is guaranteed to converge by observing a single point and having an unknown object motion. In addition, for a more accurate treatment, this paper extensively validates this approach for both static and dynamic object in the presence of measurement noise.

The remainder of this paper is organized as follows: Necessary preliminaries and state dynamics formulation are sought in Section 2. Section 3 presents the design of the Nonlinear Unknown Input Observer NLUIO to estimate structure of a feature point where LMI-based formulation is developed to prove asymptotic convergence. In Section 4 the simulation results are demonstrating the robustness of the approach in the presence of measurement noise. Finally, concluding remarks are drawn in Section 5.

\section{STATE DYNAMIC FORMULATION}

In this section an overview of the perspective relationships and basic kinematic is given modeling a camera which moves and observes a moving object. Most of the concepts can be found, for example, in [21] and [26]. Consider a scenario in Figure 1 where the motion of a single moving object is viewed by a moving camera undergoing rotation and translation. The equation of a feature point in the object can be presented in the reference frame as

$$
\dot{x}=[\omega] \cdot x+v
$$

Where, the state vector $x(t)=\left[\begin{array}{lll}x_{1}(t) & x_{2}(t) & x_{3}(t)\end{array}\right]^{T} \in \square^{3}$ is defined rather as $x=[X / Z, Y / Z, 1 / Z]^{T}$, With : $X, Y$ and $Z$ are the unknown Euclidean coordinates of feature point in the camera's inertial frame. $x_{3}(t)$ being perpendicular to the camera's image plane is the inverse of an unmeasurable focal distance. 
$[\omega] \in \square^{3 \times 3}$ represent a skew symmetric matrix made from the angular motion of the moving camera $\omega(t)=\left[\begin{array}{lll}\omega_{1}(\mathrm{t}) & \omega_{2}(\mathrm{t}) & \omega_{3}(t)\end{array}\right]^{T}$ and given by

$$
[\omega]=\left[\begin{array}{ccc}
0 & -\omega_{3} & \omega_{2} \\
\omega_{3} & 0 & -\omega_{1} \\
-\omega_{2} & \omega_{1} & 0
\end{array}\right]
$$

$v(t)$ Is a relative camera linear velocity, such that $v=v_{c}-v_{p}$, where $v_{c}=\left[\begin{array}{lll}v_{c 1} & v_{c 2} & v_{c 3}\end{array}\right]^{T}$ is the linear camera velocity and $v_{p}=\left[\begin{array}{lll}v_{p 1} & v_{p 2} & v_{p 3}\end{array}\right]^{T}$ is the feature point velocity. Note that velocities $v_{c}$ and $v_{p}$ are bounded and continuously differentiable.

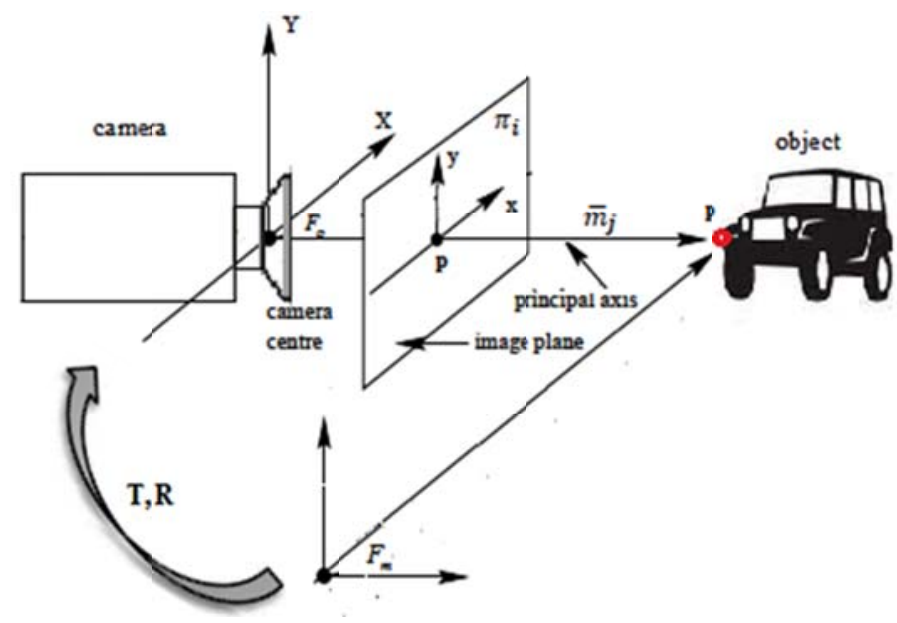

Figure 1. Camera object motion model

The next development aimed to design of NLUIO observers is to estimate the structure of moving object with respect to a moving camera. The relative motion of the feature point can be given by substituting $\omega(t)$ and $v(t)$ into (1) as follows

$$
\begin{aligned}
& \dot{x}_{1}=-x_{1} x_{2} \omega_{1}+\omega_{2}+x_{1}^{2} \omega_{2}-x_{2} \omega_{3}+\left(v_{c 1}-x_{1} v_{c 3}\right) x_{3}-v_{p 1} x_{3}+x_{1} v_{p 3} x_{3} \\
& \dot{x}_{2}=-\omega_{1}-x_{2}^{2} \omega_{1}+x_{1} x_{2} \omega_{2}+x_{1} \omega_{3}+\left(v_{c 2}-x_{2} v_{c 3}\right) x_{3}-v_{p 2} x_{3}+x_{2} v_{p 3} x_{3} \\
& \dot{x}_{3}=-v_{c 3} x_{3}^{2}-\left(x_{2} \omega_{1}-x_{1} \omega_{2}\right) x_{3}+v_{p 3} x_{3}^{2} \\
& y=\left[\begin{array}{ll}
x_{1} & x_{2}
\end{array}\right]^{T}
\end{aligned}
$$

The equations above are composed of unmeasurable coordinate $x_{3}$ and unknown motion information of the moving scene. To recuperate the 3D structure, the state vector should be estimated. That's why the unknown motion parameters of both camera and object are separated in the following. We define

$$
\begin{aligned}
& G_{1}(u, y) \square-x_{1} x_{2} \omega_{1}+\omega_{2}+x_{1}^{2} \omega_{2}-x_{2} \omega_{3} \\
& G_{2}(u, y) \square-\omega_{1}-x_{2}^{2} \omega_{1}+x_{1} x_{2} \omega_{2}+x_{1} \omega_{3} \\
& F_{1}(x, u) \square\left(v_{c 1}-x_{1} v_{c 3}\right) x_{3} \\
& F_{2}(x, u) \square\left(v_{c 2}-x_{2} v_{c 3}\right) x_{3} \\
& F_{3}(x, u) \square-v_{c 3} x_{3}^{2}-\left(x_{2} \omega_{1}-x_{1} \omega_{2}\right) x_{3}
\end{aligned}
$$




$$
\begin{aligned}
& D_{1} \square-v_{p 1} x_{3}+x_{1} v_{p 3} x_{3} \\
& D_{2} \square-v_{p 2} x_{3}+x_{2} v_{p 3} x_{3} \\
& D_{3} \square v_{p 3} x_{3}^{2}
\end{aligned}
$$

With $G_{2}(u, y), F_{1}(x, u), F_{2}(x, u), F_{3}(x, u), d_{1}(x), d_{2}(x)$ and $d_{3}(x) \in \square$ and $u=\left[\begin{array}{ll}v_{c} & \omega\end{array}\right]^{T}$ is a measurable input constituted by the angular and the linear camera velocities. Consequently, the dynamics in (3) can be rewritten in nonlinear system form as follows

$$
\left\{\begin{array}{l}
\dot{x}=f(x, u)+g(y, u)+H d(t) \\
y=C x
\end{array}\right.
$$

Where $x(t) \in \square^{n}$ is the state of the normalized Euclidean coordinates, $d(t) \in \square^{q}$ is an unknown input which contains the point velocities, $y(t) \in \square^{p}$ the output, $g(y, u) \in \square^{3}$ and $f(x, u): \square^{3} \rightarrow \square$ are nonlinear functions. $H \in \square^{n \times q}$ is the unknown input matrix and $C \in \square^{p \times n}$ is the output matrix.

Remark 1 (Observability) [27]: The nonlinear system in (4) is not observable if all the linear and angular velocities of the camera are null. That means $v_{c}(t)=0$ and $\omega(t)=0$. In addition, where the moving camera and the feature point of the moving object follow the same ray, it means that $v_{c 1}-v_{p 1}-x_{1}\left(v_{c 3}-v_{p 3}\right)=v_{c 2}-v_{p 2}-x_{2}\left(v_{c 3}-v_{p 3}\right)=0$

In our case, the dynamic system in (4) can be rewritten as follows

$$
\left\{\begin{array}{l}
\dot{x}=A x+\tilde{f}(x, u)+g(y, u)+H d \\
y=C x
\end{array}\right.
$$

Where $\tilde{f}(x, u)=f(x, u)-A x$ and $A \in \square^{3 \times 3}$.

Note that the nonlinear function $f(x, u)$ is a Lipschitz function [28] and $\hat{x}$ is the estimated state vector such that for the same positive constant $\alpha$ independent of $x$

$$
\|f(x, u)-f(\hat{x}, u)\| \leq \alpha\|x-\hat{x}\|
$$

When the camera is moving with faster velocities the Lipschitz constant $\alpha$ is large. As regards to the nonlinear function $\tilde{f}(x, u)$, it is also a Lipschitz function so there exists a positive Lipschitz constant $\beta$ such that

$$
\|f(x, u)-f(\hat{x}, u)-A(x-\hat{x})\| \leq(\alpha+\beta)\|x-\hat{x}\|
$$

Given an observable perspective system, the design purpose is to estimate the coordinates $x(t)$ from the linear and the angular camera velocities.

The overall structure of the proposed method is shown in Figure 2. After the state dynamic formulation, the NLUIO for the structure estimation will be achieved in the following section

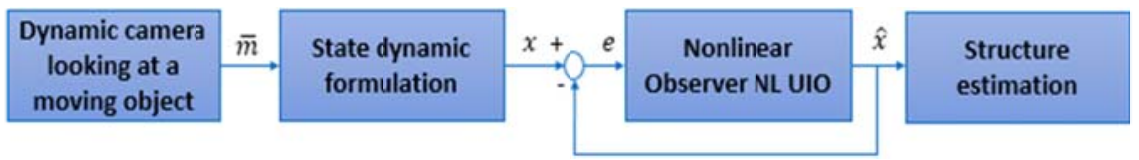

Figure 2. Structure of the proposed structure estimation method 


\section{OBSERVER FORMULATION}

In this section, an asymptotically converging NLUIO is constructed, the state of which follows the state of the dynamics system given in (5) as closely as possible further in the presence of an unknown input. For the rest of the study it is going to be assumed that the following conditions [29] are satisfied:

- $H$ is assumed to be column rank matrix

$-\operatorname{rank}(\mathrm{CH})=\operatorname{rank}(H)=q$.

Where $q$ the number of the unknown input.

With above conditions, the NLUIO for system represented by (5) can be shown as follows

$$
\left\{\begin{array}{l}
\dot{z}=N z+L y+M \tilde{f}(\hat{x}, u)+M g(y, u) \\
\hat{x}=z-E y
\end{array}\right.
$$

Where $\hat{x}(t) \in \square^{n}$ is an estimate of $x(t)$ and $z(t) \in \square^{n}$ is the state observer. Matrices $N \in \square^{n \times n}, L \in \square^{n \times p}$, $E \in \square^{n \times p}$ and $M \in \square^{n \times n}$ are determined to design the observer such that $x(t)$ eventually tends to $\hat{x}(t)$ in the face of unknown input. Observer gain matrices equations can be expressed in alternate form

$$
\left\{\begin{array}{l}
N<0 \\
M=I+E C \\
N=M A-K C \\
L=K(I+C E)-M A E
\end{array}\right.
$$

Where $K$ and $E$ are gain matrices of suitable dimensions subsequently designed.

The error equation for system (5) and NLUIO (8) is defined as follows

$$
e(t)=\hat{x}(t)-x(t)=z-E y-x=z-M x
$$

By substituting the system output presented in (5) into the error equation in (10), the dynamic error $\dot{e}(t)$ will have the following form

$$
\dot{e}(t)=\dot{z}-(I+E C) \dot{x}
$$

Then substituting (5) and (8) into (11), the dynamic error can be expressed as follows

$$
\begin{aligned}
& \dot{e}=N e+N(I+E C) x+L C x+M(\tilde{f}(\hat{x}, u)-\tilde{f}(x, u))-M A x-M H d \\
& \dot{e}=N e+(N M+L C-M A) x+M(\tilde{f}(\hat{x}, u)-\tilde{f}(x, u))-M H d
\end{aligned}
$$

To obtain matrices, the following steps should be followed: First using (9) the equation $N M+L C-M A=0$ is satisfied, and if the matrix $E$ satisfies (13)

$$
M D=(I+E C) D=0
$$

Then the equation of the error dynamics in (12) yields to

$$
\dot{e}=N e+M(\tilde{f}(\hat{x}, u)-\tilde{f}(x, u))
$$

The condition in (13) can be written as

$$
E C H=-H
$$

After that, a solution exists for matrix $E$ using generalized inverse as follows

$$
\begin{aligned}
& E=-H(C H)^{+}+Y\left(I_{q}-(C H)(C H)^{+}\right) \\
& E=F+Y G
\end{aligned}
$$


Where $Y \in \square^{n \times q}$ an arbitrary matrix, $F=-H(C H)^{+}$and $G=\left(I_{2}-(C H)(C H)^{+}\right)$.

Finally, by substituting $E$ into (9) the only unknowns are matrices $K$ and $Y$. The following section presents a theorem that gives a sufficient condition for choosing them.

\subsection{LMI sufficient condition}

Theorem: The error $e(t)$ will converge asymptotically to 0 for any initial value $e(0)$ and the NLUIO in (8) is exponentially stable such that $\|e(t)\| \leq\left\|e\left(t_{0}\right)\right\| \exp (-\lambda t)$, where $\lambda \in \square^{+}$, if there exists $P$ a positive symmetric matrix $P>0$ satisfying the following condition [8]

$N^{T} P+P N+\left(\alpha^{2}+\beta^{2}\right) P M M^{T} P+2 I<0$

Proof: Let’s define the Lyapunov function candidate $V: \square^{3} \rightarrow \square$ as follows

$$
V=e^{T} P e
$$

This Lyapunov function verify the inequality below

$$
\lambda_{\text {min }}(P)\|e\|^{2} \leq V \leq \lambda_{\text {max }}(P)\|e\|^{2}
$$

Where $\lambda_{\min }$ and $\lambda_{\max }$ are the minimum and the maximum Eigen values of $P$. By expanding the Lyapunov candidate function of (18) along the error equation in (14) the following expression is obtained

$$
\begin{aligned}
& \dot{V}=e^{T}\left(N^{T} P+P N\right) e+2 e^{T} P M(\tilde{f}(\hat{x}, u)-\tilde{f}(x, u)) \\
& \dot{V}=e^{T}\left(N^{T} P+P N\right) e+2 e^{T} P M(f(\hat{x}, u)-f(x, u))-2 e^{T} P M A(\hat{x}-x) \\
& \dot{V} \leq e^{T}\left(N^{T} P+P N\right) e+2\left\|e^{T} P M\right\|\|A\|\|e\|+2\left\|e^{T} P M\right\|\|(f(\hat{x}, u)-f(x, u))\| \\
& \dot{V} \leq e^{T}\left(N^{T} P+P N\right) e+2\left\|e^{T} P M\right\| \alpha\|e\|+2\left\|e^{T} P M\right\| \beta\|e\|
\end{aligned}
$$

then using the bellow inequality, where $\alpha \in \square^{+}$and $\beta \in \square^{+}$.

$$
2 \alpha\left\|e^{T} P M\right\|\|e\| \leq \alpha^{2}\left\|e^{T} P M\right\|^{2}+\|e\|^{2} \text { And } 2 \beta\left\|e^{T} P M\right\|\|e\| \leq \beta^{2}\left\|e^{T} P M\right\|^{2}+\|e\|^{2}
$$

after simplification, $\dot{V}$ may be reconstructed as

$$
\begin{aligned}
& \dot{V} \leq e^{T}\left(N^{T} P+P N\right) e+\left(\alpha^{2}+\beta^{2}\right) e^{T} P M M^{T} P e+2 e^{T} e \\
& \dot{V} \leq e^{T}\left(N^{T} P+P N+\left(\alpha^{2}+\beta^{2}\right) P M M^{T} P+2 I\right) e
\end{aligned}
$$

Define the matrix $Q<0$ by $Q=N^{T} P+P N+\left(\alpha^{2}+\beta^{2}\right) P M M^{T} P+2 I$, hence the time derivative $\dot{V}$ is presented as

$$
\dot{V} \leq e^{T} Q e
$$

Using (19) and (20), the upper bound for $V(t)$ can be written as $V(t) \leq V\left(t_{0}\right) \exp (-\lambda t)$ where $\lambda=\frac{\lambda_{\max }(Q)}{\lambda_{\text {min }}(P)}$ and the upper bound for $e(t)$ is expressed by $\|e(t)\| \leq \varphi\left\|e\left(t_{0}\right)\right\| \exp (-\lambda t)$ where $\varphi=\lambda_{\max }(P) / \lambda_{\min }(P)$.

The condition rank $\left[\begin{array}{cc}s I_{n}-A & D \\ C & 0\end{array}\right]=n+q, \forall s \in \square$ leads to the fact that $(M A, C)$ is observable. In consequence, the matrix $K$ can be obtained such that $N=M A-K C$ is Hurwitz equality. 
Note that there is no systematic way to obtain the adaptable NLUIO parameters directly from condition (10) and the expression in the theorem given by (17). This allows to reformulate them as LMIs. Substituting $N$ and $M$ from (9) into (17) the following relationship can then be established

$$
(M A-K C) P+P(M A-K C)+2 I+\left(\alpha^{2}+\beta^{2}\right) P(I+E C)(I+E C)^{T} P<0
$$

Using the solution in (16), the inequality (21) becomes

$$
\begin{aligned}
& A^{T}(I+F C)^{T} P+P(I+F C) A+A^{T} C^{T} G^{T} P_{1}^{T}+P_{1} G C A-C^{T} P_{2}^{T}-P_{2} C \\
& +2 I+\left(\alpha^{2}+\beta^{2}\right)\left(P+P F C+P_{1} G C\right)\left(P+P F C+P_{1} G C\right)^{T}<0
\end{aligned}
$$

Variables $P_{1}=P Y$ and $P_{2}=P K$ are given to make the resolution of the nonlinear matrix inequalities easier. Exponential convergence to the object coordinates is achieved.

\subsection{LMI formulation}

For the NLUIO synthesis the following LMIs (23) have feasible solutions for P, K and Y invoking the inequality in (22) transformed with schur's complement.

$$
\left[\begin{array}{cc}
X & \gamma W \\
\gamma W^{T} & -I
\end{array}\right]<0
$$

Where $\quad W=\left(P+P F C+P_{1} G C\right)$,

$$
\begin{aligned}
& X=A^{T}(I+F C)^{T} P+P(I+F C) A+A^{T} C^{T} G^{T} P_{1}^{T}+P_{1} G C A-C^{T} P_{2}^{T}-P_{2} C+2 I \\
& \gamma=\left(\alpha^{2}+\beta^{2}\right) .
\end{aligned}
$$

Using solution of LMI i.e. feasible values of $Y=P^{-1} P_{1}$ and $K=P^{-1} P_{2}$, observer matrices satisfying the requisite conditions are found. The LMI feasibility can be solved using standard LMI approach [30].

\section{RESULTS AND DISCUSSIONS}

In contrast with previous research that assume noise-free measurements and demand prior knowledge of the object and camera motion, the proposed method assume that the object velocity is unknown. In the following, the performance of the NLUIO is validated through different numerical simulations in the presence of measurement noise for both static and dynamic scenes. As the current simulation results are restricted to tracking a single point feature. Two different object motion models are considered, and the proposed NLUIO performance is evaluated for both cases. Whereas the usual speed of the monocular camera is 30 frames/s, the NLUIO is valid for a continuous-time system. For the simulation results, SIMULINK is used with sampling period $t_{s}=10^{-3} \mathrm{~s}$. The initial Euclidean coordinates of the object feature are $x\left(t_{0}\right)=\left[\begin{array}{lll}5 & 2 & 1\end{array}\right]^{T}(\mathrm{~m})$. Since initial target feature point is not known at the NLUIO, thus the system and observer start from different initial conditions. Initial condition for the observer is taken as $\hat{x}\left(t_{0}\right)=\left[\begin{array}{lll}-1 & -0.5 & -0.2\end{array}\right]^{T}(\mathrm{~m})$.

Matrices $A, C$ and $H$ are given by

$$
A=\left[\begin{array}{ccc}
0 & -1 & 2 \\
1 & 0 & 1 \\
0 & 0 & 0
\end{array}\right] C=\left[\begin{array}{lll}
1 & 0 & 0 \\
0 & 1 & 0
\end{array}\right] \text { And } D=\left[\begin{array}{lll}
1 & 0 & 0
\end{array}\right]^{T}
$$

Note, the third component $x_{3}$ of the state, which is the unmeasurable distance between the camera and the moving object. Clearly the estimation of the three-dimensional Euclidean coordinates can yield the distance estimation. The comparison of RMS error values obtained with the proposed NLUIO with different values of measurement noise was used to demonstrate the proposed method. 


\subsection{Static scene}

In this case, the object and the camera velocities parameters are chosen respectively by

$$
v_{c}=\left[\begin{array}{c}
1 \\
2 \\
0.2 \sin (\pi t / 4)
\end{array}\right](\mathrm{m} / \mathrm{s}), \omega=\left[\begin{array}{lll}
0 & 0 & -\pi / 30
\end{array}\right]^{T}(\mathrm{rad} / \mathrm{s}) \text { and } v_{p}=\left[\begin{array}{c}
0.5 \\
0 \\
0
\end{array}\right](\mathrm{m} / \mathrm{s})
$$

Figure 3 shows the structure estimation of the object position in the single camera images. Figure 4 presents the error in the position estimation of the static object. Notice that the transient performance of the proposed scheme is significantly less then 4 second. The RMS error values obtained by the proposed NLUIO are given as follows

$$
\mathrm{e}_{1}=0.1578, \mathrm{e}_{2}=0.0789 \text { and } \mathrm{e}_{3}=0.0776 \text {. }
$$
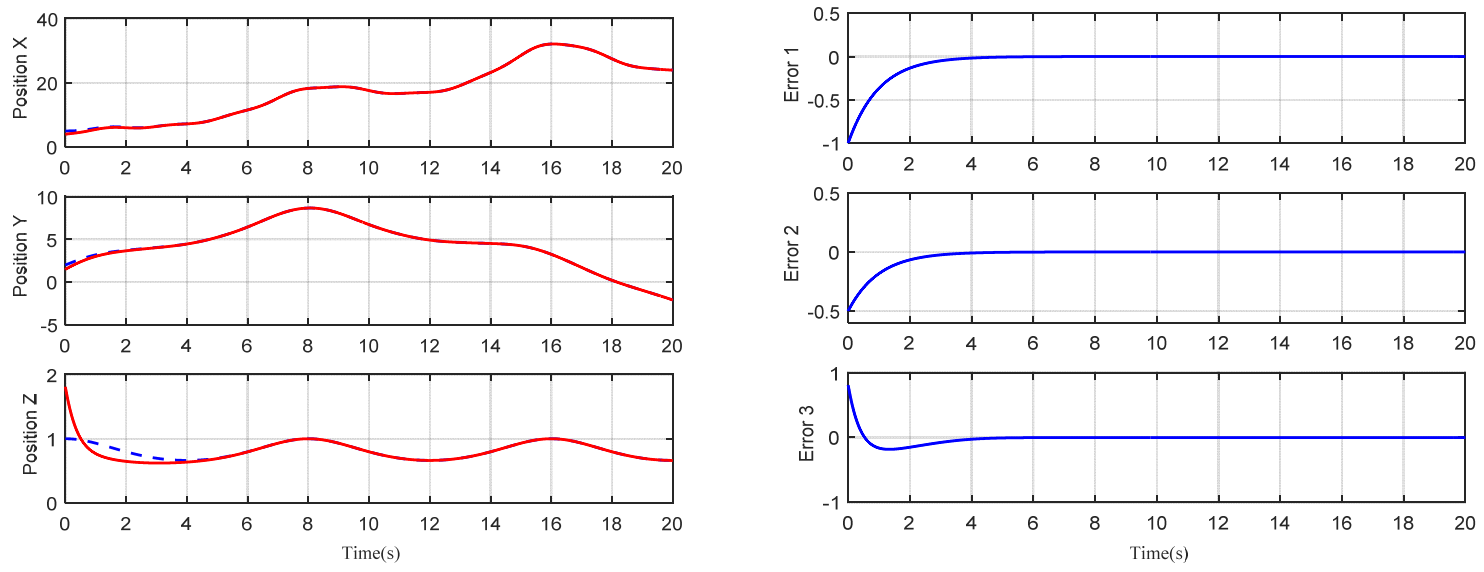

Figure 3. Time histories of the static object position in the single-camera images; solid line: estimated and Dashed-dotted line: real state

Figure 4. Static object estimation error of the proposed method

Next, the measurements of $V_{c}$ as shown in Figure 5 is assumed to be corrupted by adding a Band Limited White Noise (BLWGN) with $5 \%$ of power, a correlation time of 0 and a covariance of infinity. Figure 6 shows the structure estimation of the static object position in the single camera images in the presence of measurement noise. The error in the position estimation of the static object is described in Figure 7. Only the third component of RMS error is changed $e_{3}=0.0816$.
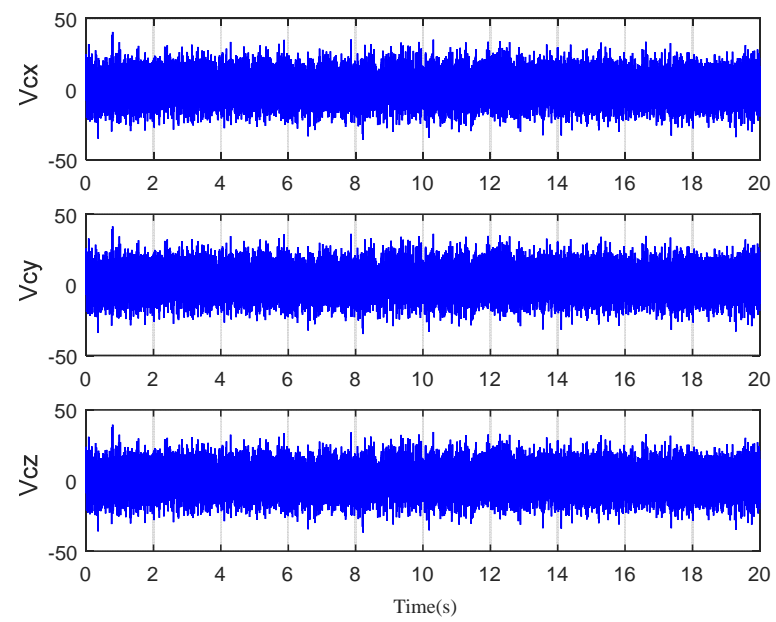

Figure 5. The measurments of the camera velocities in the presence of noise 

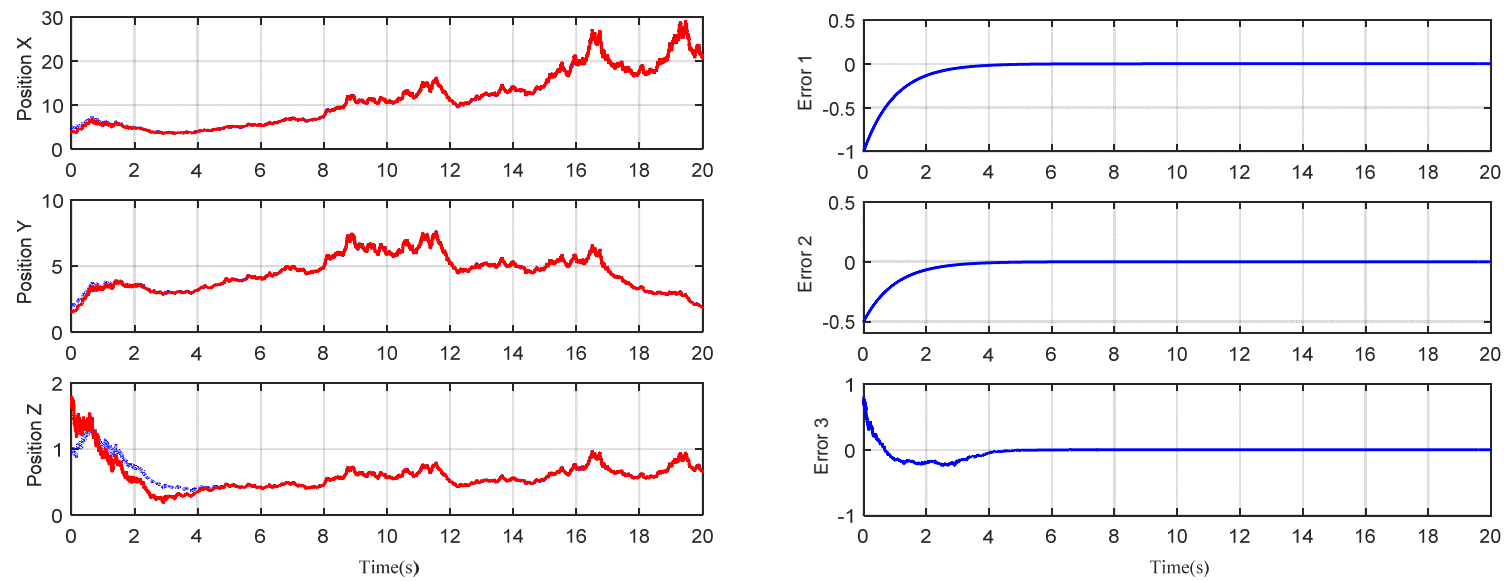

Figure 6. Time histories of the static object position in the single-camera images in the presence of noisy camera velocity; solid line: estimated and Dashed-dotted line: real state

Figure 7. Static object estimation error of the proposed method in the presence of noisy camera velocity

In addition to the previous measurement noise of $V_{c}$, the proposed observer is validated for robustness by the addition of a Band Limited White Noise (BLWGN) with $5 \%$ of power to the object velocity. Figure 8 shows the structure estimation of the dynamic object coordinates with noisy object and camera velocities and Figure 9 describes the error in the position estimation of the dynamic. The NLUIO then yields uniformly asymptotically convergent estimates of the three-dimensional Euclidean coordinates of the feature point. In the presence of noise in the motion parameters, the estimated state $\hat{x}_{3}$ is corrupted directly by the source of noise, therefore the third component of RMS error increases and becomes $\mathrm{e}_{3}=0.2048$.
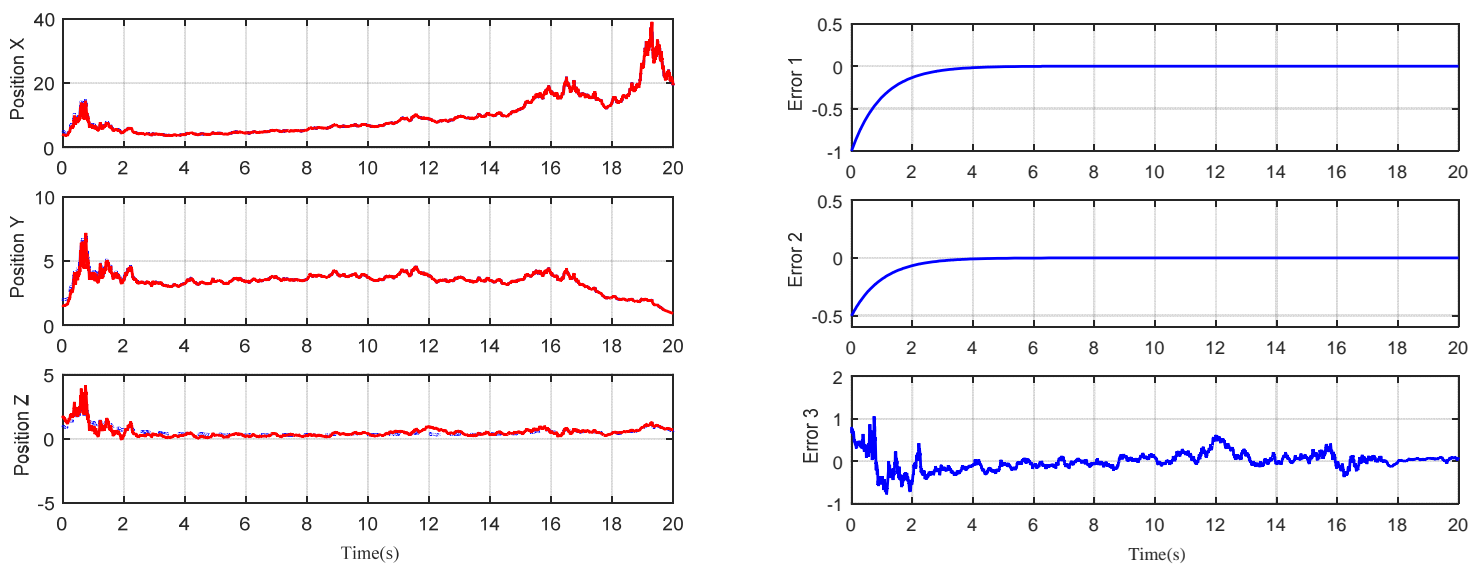

Figure 8. Time histories of the static object position in the single-camera images in the presence of noisy camera and object velocities; solid line: estimated and

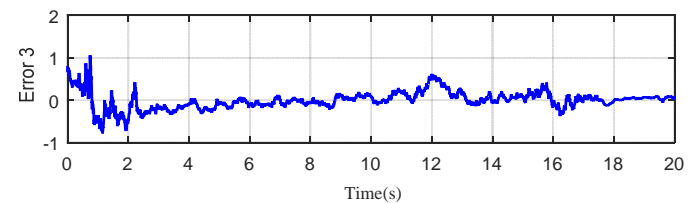

Figure 9. Static object estimation error of the proposed method in the presence of noisy camera and object velocities

Dashed-dotted line: real state

\subsection{Dynamic scene}

In this case, only the performance in the presence of measurement noise for both camera and object velocity is studied. The same values of measurement noise are used. The object and the camera velocities are chosen respectively as: 
$v_{c}=\left[\begin{array}{c}1 \\ 2 \\ 0.2 \sin (\pi t / 4)\end{array}\right](m / s), \omega=\left[\begin{array}{lll}0 & 0 & -\pi / 30\end{array}\right]^{T}(\mathrm{rad} / \mathrm{s})$ and $v_{p}=\left[\begin{array}{c}0.2+\cos (2 \pi \mathrm{t} / 4) \\ 0 \\ 0\end{array}\right](\mathrm{m} / \mathrm{s})$.

Figure 10 presents the structure estimation of the dynamic object position and Figure 11 shows the error in the position estimation of the object with noisy camera velocity. These results demonstrate that the proposed NLUIO based object structure estimation method can achieve satisfactory performance even with camera velocities. This observer gives better estimates for a significant level of noise even changing scene. RMS error values are given as follows:

$\mathrm{e} 1=0.1578, \mathrm{e} 2=0.0789$ and $\mathrm{e} 3=0.0816$.
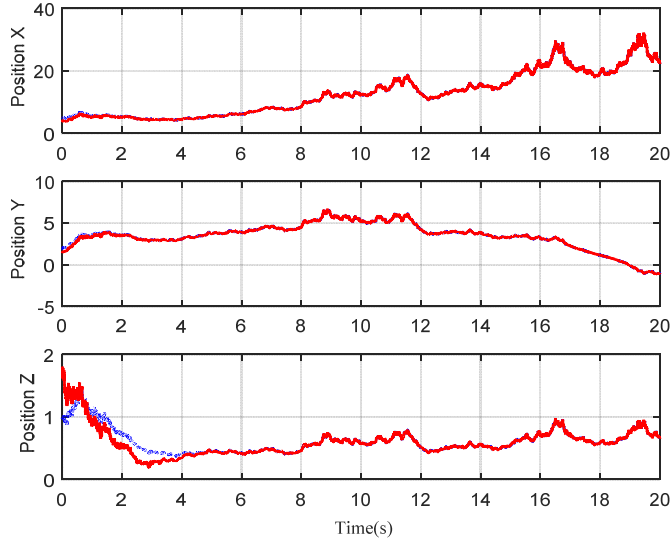

Figure 10. Time histories of the dynamic object position in the presence of noisy camera velocity; solid line: estimated and Dashed-dotted line: real state
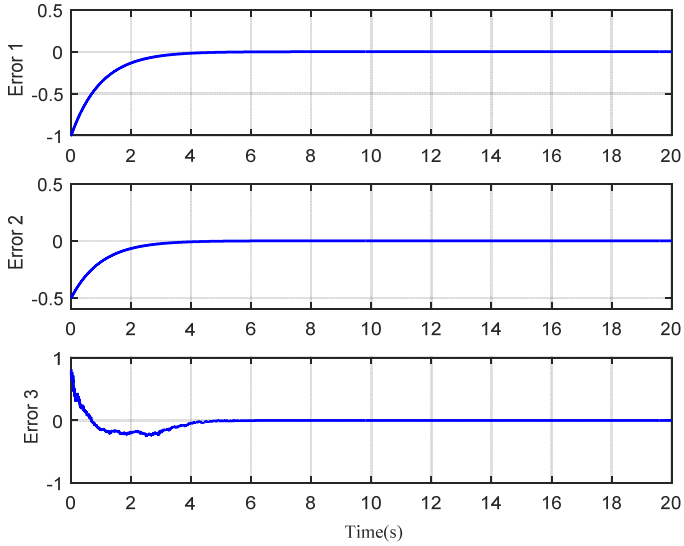

Figure 11. Dynamic object estimation error of the proposed method in the presence of noisy camera velocity

Figure 12 shows the structure estimation of the dynamic object position with noisy object and camera velocities and Figure 13 shows the structure estimation error of the dynamic object. Here again, only the third component of RMS error are changed $e_{3}=0.0992$. However, the presence of noise on both camera and object velocities can significantly degrade the performance of NLUIO. Therefore, it can be seen that the practical situation does require a more robust nonlinear observer for the considered problem.
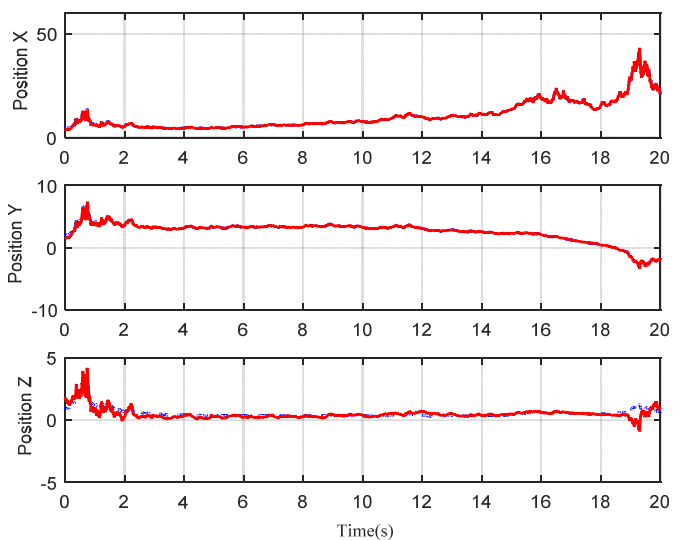

Figure 12. Time histories of the dynamic object position in the presence of noisy camera and object velocities; solid line: estimated and Dashed-dotted line: real state
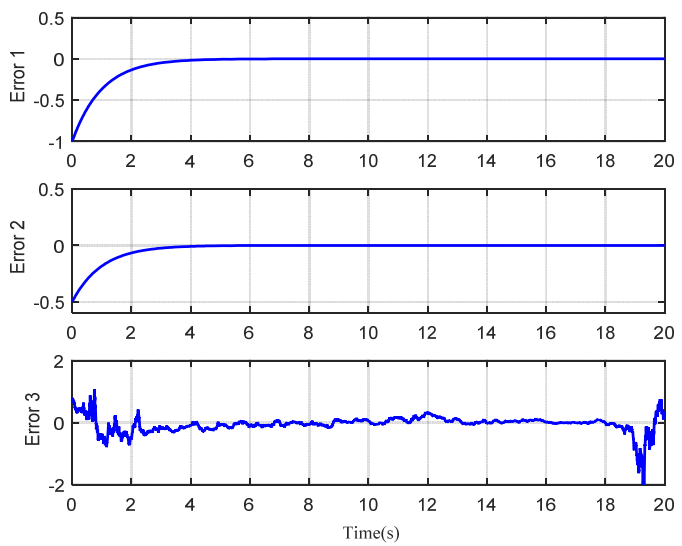

Figure 13. Dynamic object estimation error of the proposed method in the presence of noisy camera and object velocities 


\section{CONCLUSION}

The robust NLUIO has been designed for a nonlinear camera-object system. Furthermore, the stability of the error systems has been demonstrated to estimate structure from motion of a feature point. The sufficient condition for existence of the designed nonlinear observer is derived. In order to facilitate the NLUIO design, the obtained condition is formulated in terms of LMIs. This paper extensively validates the proposed method for both static and dynamic scenes. Simulation results are promising and much has to be done to assess the performance of the proposed method against measurement noise. An interesting direction for future research is improving the proposed method to test it with experimental data and considering a trajectory of a moving object along a plane.

\section{REFERENCES}

[1] S. El Hazzat, M. Merras, N. El Akkad, A. Saaidi, K. Satori, “3D reconstruction system based on incremental structure from motion using a camera with varying parameters,” The Visual Computer, vol. 34, no. 10, pp. 1443-1460, Oct 2018.

[2] Q. Xu, J. Li, W. Tao, D. Ming, “Efficient large-scale geometric verification for structure from motion,” Pattern Recognition Letters, vol. 125, pp. 166-173, 2019.

[3] M. Tabesh, T. Hoffmann, S. Vollmer, H. Schüttrumpf, R. M. Frings, "In-situ measurement of river-bed sediment porosity using Structure-from-Motion image analysis,” Geomorphology, vol. 338, pp. 61-67, 2019.

[4] Y. Guo, “A minimal solution for plane motion and structure from two perspective views," Pattern Recognition Letters, vol. 120, pp. 96-103, 2019.

[5] X. Wang, F. Rottensteiner and C. Heipke, "Structure from motion for ordered and unordered image sets based on random k-d forests and global pose estimation,” ISPRS Journal of Photogrammetry and Remote Sensing, vol. 147, pp. 19-41, 2019.

[6] M. Shahbazi, P. Ménard, G. Sohn, J. Théau, “Unmanned aerial image dataset: Ready for 3D reconstruction,” Data in Brief, vol. 25, 2019.

[7] K. Anderson and K.J Gaston, “Light weight unmanned aerial vehicles will revolutionize spatial ecology,” Front. Ecol. Environ, vol. 11, pp.138-146, 2014.

[8] D. Ventura, M. Bruno, G.J. Lasinio, A. Belluscio and G.A. Ardizzone, "low-cost drone-based application for identifying and mapping of coastal fish nursery grounds,” Estuar. Coast. Shelf Sci., vol. 171, pp. 85-98, 2016.

[9] V. Chirayath and S.A. Earle, "Drones that see through waves-preliminary results from airborne fluid lensing for centimetre-scale aquatic conservation,” Aquat. Conserv. Mar. Freshw. Ecosyst, vol. 26, pp. 237-250, 2016.

[10] J. A. Morgan, D. J. Brogan and P. A. Nelson, “Application of Structure-from-Motion photogrammetry in laboratory flumes,” Geomorphology, vol. 276, pp. 125-143, Jan 2017.

[11] C. D. Carlton, S. Mitchell and P. Lewis, "Preliminary application of Structure from Motion and GIS to document decomposition and taphonomic processes,” Forensic Science International, vol. 282, pp. 41-45, 2018.

[12] L. A. Méndez-Barroso, J. L. Zárate-Valdez and A. Robles-Morúa, "Estimation of hydromorphological attributes of a small forested catchment by applying the Structure from Motion (SfM) approach," International Journal of Applied Earth Observation and Geoinformation, vol. 69, pp. 186-197, 2018.

[13] C. H. De Souza, R. A. Lamparelli, J. V. Rocha and P. S. Magalhães, "Height estimation of sugarcane using an unmanned aerial system (UAS) based on structure from motion (SfM) point clouds," International Journal of Remote Sensing, vol. 38, pp. 2218-2230, 2017.

[14] A. De Luca, G. Oriolo, P. R. Giordano, "Feature Depth Observation for Image-based Visual Servoing: Theory and Experiments,” The International Journal of Robotics Research, vol. 27, no. 10, pp. 1093-1116, Oct 2008.

[15] A. Dani, Z. Kan, N. R. Fischer, and W. E. Dixon, "Structure and motion estimation of a moving object using a moving camera,” Proc. Am. Control Conf., pp. 6962-6967, 2010.

[16] A. Dani, Z. Kan, N. R. Fischer, and W. E. Dixon, "Structure estimation of a moving object using a moving camera: An unknown input observer approach,” IEEE Conference on Decision and Control and European Control Conference (CDC-ECC), pp. 5005-5010, 2011.

[17] A.P. Dani, N.R. Fischer, Z. Kan, and W.E. Dixon, "Globally exponentially stable observer for vision-based range estimation,” Mechatronics, vol. 22, pp. 381-389, 2012.

[18] I. Grave and Y. Tang, “A New Observer for Perspective Vision Systems under Noisy Measurements,” IEEE Transactions on Automatic Control, vol. 60, no. 02, pp. 503-508, Feb 2015.

[19] M. Unel, A. Sabanovic, B. Yilmaz and E. Dogan, Visual motion and structure estimation using sliding mode observers, International Journal of Systems Science, vol. 39, no. 2, pp. 149-161, 2008.

[20] A. P. Dani, N. R. Fischer and W. E. Dixon, "Single Camera Structure and Motion," IEEE Transactions on Automatic Control, vol. 57, no. 01, pp. 238-243, Jan 2012.

[21] D. Chwa, A. P. Dani and W. E. Dixon, "Range and Motion Estimation of a Monocular Camera Using Static and Moving Objects,” IEEE Transactions on Control Systems Technology, vol. 24, no. 04, pp. 1174-1183, Jul 2016.

[22] D. Chwa, A. Dani, H. Kim and W. Dixon, "Camera motion estimation for 3-D structure reconstruction of moving objects,” 2012 IEEE International Conference on Systems, Man, and Cybernetics (SMC), Seoul, pp. 1788-1793, 2012.

[23] H. Xie, R. T. Fomena, and A. F. Lynch, "Reduced-order Observer Design for Structure and Motion Estimation," 2013 Proceedings of the Conference on Control and its Applications, California, pp. 37-43, 2013. 
[24] A. Agudo, F. Moreno-Noguer, B. Calvo and J. M. M. Montiel, Sequential Non-Rigid Structure from Motion Using Physical Priors, IEEE Transactions on Pattern Analysis and Machine Intelligence, vol. 38, no. 05, pp. 979-994, May 2016.

[25] J. Keshavan and J.S. Humbert, "Robust structure and motion recovery for monocular vision systems with noisy," measurements, International Journal of Control, vol. 91, no. 03, pp. 715-724, 2018.

[26] Z. Mejri, L. Sidhom and A. Abdelkrim, "Structure recovering of moving object using a real time approach," 2018 International Conference on Advanced Systems and Electric Technologies (IC_ASET), Hammamet, 2018, pp. 66-71.

[27] R. Hermann and A. Krener, "Nonlinear controllability and observability," IEEE Transactions on Automatic Control, vol. 22, no. 5, pp. 728-740, Oct 1977.

[28] H.K. Khalil, Nonlinear systems. Prentice Hall, $3^{\text {rd }}$ ed., 2002.

[29] M. Darouach, M. Zasadzinski, and S. Xu, “Full-order observers for linear systems with unknown inputs,” IEEE Transactions on Automatic Control, vol. 39, pp. 606-609, 1994.

[30] P. Gahinet, A. Nemirovskii, A. Laub, and M. Chilali, “The LMI control toolbox,” IEEE Conf. Decision and Control, vol. 3, pp. 2038-2041, 1994.

\section{BIOGRAPHIES OF AUTHORS}
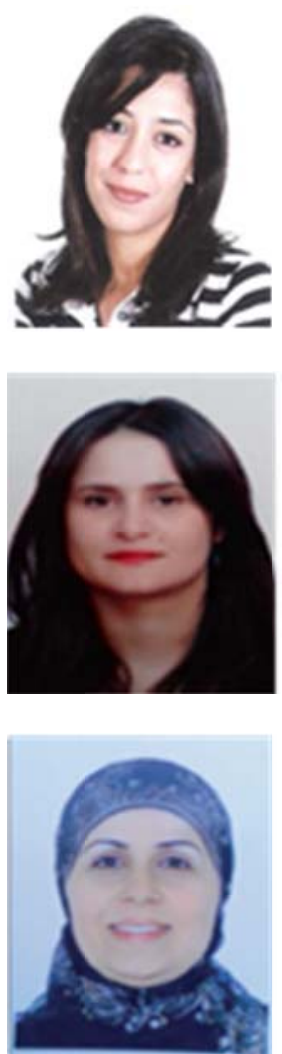

Zoubaida Mejri was born in Tunisia, in 1984. She received the electrical engineering degree in 2009 from Higher School of Technology and Informatics (ESTI) transformed now to the National Engineering School of Carthage (ENICarthage) and a Master Diploma in the Automatic and Industrial Computing from the National Institute of Applied Sciences and Technology (INSAT) in 2012. She is a PhD student with the Researclh Laboratory in Automatic Control (LARA), National Engineering School of Tunis (ENIT). Her current research interests electrical, automatic control, sliding mode, observer/estimator and computer vision.

Lilia Sidhom was born in Nabeul, in 1982. She received the Automatic and industrial computing degree from the National Institute of Applied Sciences and Technology (INSAT) Tunis in 2006 and the M.Sc. and PhD degrees in automatic from the National Institute of Applied Sciences of Lyon (INSA Lyon), France, in 2007 and 2011, respectively. She is an Assistant Professor at National Engineering School of Bizerte, since September 2016. Her" current research interests include advanced control of Fluid Power and mechatronics systems, in soft sensor, fault detection, higher sliding modes, observer/estimator, and renewable energies.

Afef Abdelkrim was born in Tunisia in 1976. She obtained Engineer Diploma in 2000 and the PhD at the National Engineering School of Tunis (ENIT) in 2005; she is now a Professor at the National Engineering School of Carthage (ENICarthage). Her research interests are in handwriting process, handwriting process modeling and synthesis, fuzzy logic control, the artificial neuronal network domain and in deep learning. 